\title{
VERANTWORTUNG VON UNTERNEHMEN \\ BEI VERLETZUNG DER PRIVATSPHÄRE - VERANTWORTUNG VON UND IN NETZWERKEN
}

\section{CSENGE HALÁSZ*}

\begin{abstract}
Certain technological improvements mean the obvious rethinking of the boundaries of the private sphere. This process has made a legislative response necessary. In this sense, Act LIII of 2018 can be highlighted, which states in its preamble that the modern devices of info communication have laid new foundation for the everyday communication, hence the protection of private life extends beyond the physical harassment to Internet. An important factor in the protection of privacy is the responsibility of companies, which must ensure the protection of the privacy rights of entities using their online interfaces.
\end{abstract}

Keywords: the right to privacy, personal rights, responsibility, social media

\section{EiNLEITUNG}

Die verschiedenen sozialen Netzwerke haben zwischenmenschliche Beziehungen zwischen Einzelpersonen eindeutig verändert. Vielleicht ist es überflüssig 2020 darüber zu sprechen, da wir die Auswirkungen der neuen Online-Schnittstellen hautnah miterleben, was sich auch an der Anzahl der Benutzer zeigt. Der Marktführer Facebook wird von mehr als zwei Milliarden Menschen weltweit genutzt, von denen mehr als fünf Millionen inländische (ungarische) Nutzer sind. ${ }^{1}$ Dicht gefolgt von Facebook ist jedoch die Plattform YouTube, die im Jahr 2020 bereits 1,9 Milliarden aktive Nutzer hatte und oft als das „Fernsehen der Zukunft“ bezeichnet wird. Instagram ist ebenfalls eine der führenden sozialen Netzwerke, das in der Altersgruppe der 16- bis 25-Jährigen an Beliebtheit gewinnt und zu einem unverzichtbaren Werkzeug für das Online-Marketing wurde. ${ }^{2}$

Die möglichen Auswirkungen dieser Plattformen werden in einer Reihe von Gebieten angesprochen. Aus rechtlicher Sicht sollte betont werden, dass sie sich praktisch auf alle Rechtsbereiche auswirken. Eines ihrer wichtigsten Unterscheidungsmerkmale ist daher die Komplexität.

* PhD Student. Universität Miskolc, Staats- und Rechtswissenschaftliche Fakultät, Institut für Zivilwissenschaften, Lehrstuhl für Zivilrecht.

1 Top 20 Facebook Statistiken. https://zephoria.com/top-15-valuable-facebook-statistics/ (abgerufen am 16. 8. 2020).

2 Statistiken zu Social Media Plattformen für Marketingstrategien. https://www.digitalhungary.hu/kultura/Mutatjuk-hogy-kinek-milyen-kozossegi-media-platformot-erdemes-hasznalnia-marketingjehez-2020-ban/9283/ (abgerufen am 12. 1. 2021). 
In diesem Artikel sollen die rechtlichen Auswirkungen von sozialen Netzwerken auf die Persönlichkeitsrechte und die Frage der Haftung bei möglichen Verstößen untersucht werden, da die Anzahl der Fälle von Persönlichkeitsrechtsverletzungen in sozialen Medien in der ungarischen Rechtspraxis zugenommen hat.

Verstöße gegen Persönlichkeitsrechte in sozialen Medien können in zwei Richtungen auftreten: einerseits zwischen den Nutzern der Website, d. h. in einem Rechtsverhältnis zwischen Privatpersonen. Andererseits kann auch der Betreiber der Website selbst bei bestimmten Verstößen haften. In diesem Artikel soll letzteres untersucht werden.

In Bezug auf das Persönlichkeitsrecht soll vor allem auf das Recht auf Privatsphäre eingegangen werden. In einer Erklärung von 2011 sagte Facebook-Gründer Mark Zuckerberg, dass Privatsphäre nicht länger eine soziale Norm sei (,, Privacy is no longer a social norm " 3 ). Meiner Ansicht nach kann dieser Aussage nicht zugestimmt werden. Der Schutz der Privatsphäre ist im digitalen Zeitalter ein äußerst wichtiges Thema.

\section{GRUNDLEGENDE BEGRIFFLICHKEITEN}

Es gilt zunächst den Begriff „Social Media“ zu definieren, wobei jedoch keine allgemeine, genaue rechtliche Definition gegeben werden kann. Zhao Shanyang definiert soziale Medien als ,, benutzergenerierte Inhalte, die weit verbreitete Technologien verwenden, die die Kommunikation erleichtern, die Interaktion mit Gruppenmitgliedern und einem breiteren Publikum beeinflussen, typischerweise über das Internet oder ein mobiles Kommunikationsnetzwerk". 4

Im Allgemeinen kann dies aus meiner Sicht als Website verstanden werden, auf der das Framework vom Website-Betreiber bereitgestellt und dann von Benutzern mit sich ständig ändernden Inhalten ausgefüllt wird.

In Bezug auf soziale Medien ist jedoch zu beachten, dass in der jüngeren Vergangenheit viele Arten dieser Plattformen entstanden sind, die auch die Grundlage für ihre Differenzierung bilden können. Anhand einiger Beispiele können wir auch zwischen „klassischen“ sozialen Netzwerken, Messaging-Anwendungen und Videound Image-Sharing-Plattformen unterscheiden.

Der nächste zu untersuchende Begriff ist der Privatsphäre. Dieser ist seit 2014 im ungarischen Bürgerlichen Gesetzbuch als benanntes Persönlichkeitsrecht aufgeführt und seit 2018 durch eine separate sektorale Regelung geschützt. Es gibt jedoch auch keine allgemeine Definition der Privatsphäre. Der wissenschaftliche Ausgangspunkt ist der Artikel von Samuel Warren und Luis Brandeis in der Harvard Law Review von 1890 mit dem Titel ,, The Right to Privacy“ (,,Das Recht auf Privatsphäre ") $)^{5}$, der seitdem sowohl im kontinentalen als auch im angelsächsischen

3 Privacy is no longer a social norm. https://www.theguardian.com/technology/2010/jan/ 11/facebook-privacy (abgerufen am 18. 9. 2020).

4 ZHAO, Shanyang: Internet Use and Social Capital. In: Paper presented at the annual meeting of the American SociologicalAssociation, Marriott Hotel, Loews Philadelphia Hotel, Philadelphia, PA, 12. August 2005.

5 WARREN Samuel - BRANDEIS Luis: The Right to privacy. Harvard Law Review, 15. 12. 1890. 
Rechtssystem Gegenstand zahlreicher Versuche war, den Begriff zu überdenken. ${ }^{6}$ Einige von ihnen betrachteten die Privatsphäre als Hüter der individuellen Freiheit, zum Beispiel untersuchte Clinton Rossiter in seiner Arbeit von 1948 das Recht auf Privatsphäre als ein einheitliches Konzept und trat dafür ein, die Bedeutung des Individuums hervorzuheben. Nach seiner Ansicht ist „privacy [Privatsphäre] eine besondere Art von Unabhängigkeit, die als Versuch interpretiert werden kann, Autonomie zumindest in einigen persönlichen und spirituellen Aspekten zu gewährleisten, wenn dies angesichts des Drucks der modernen Gesellschaft notwendig ist".?

Der nächste wichtige Schritt innerhalb des wissenschaftlichen Diskurses war der 1960 veröffentlichte Aufsatz von William Prosser, der bis heute einen bestimmenden Maßstab in der einschlägigen US-Rechtsprechung darstellt. Prosser teilte die Fälle der Verletzung der Privatsphäre in vier Fallgruppen ein. Die erste Gruppe betrifft das Eindringen in die physische Individual- oder Privatangelegenheiten der Antragsteller. Die zweite Fallgruppe betrifft die Offenlegung intimer privater Tatsachen gegenüber der Öffentlichkeit, während die dritte Fallgruppe Mitteilungen betrifft, die den Antragsteller gegenüber der Öffentlichkeit falsch darstellten. Schließlich umfasste die vierte Gruppe die missbräuchliche Namensführung. ${ }^{8}$

Es sollen auch einige inländische dogmatische Ansätze erwähnt werden. Máté Dániel Szabó leitet das Konzept des Rechts auf Privatsphäre auch aus der Freiheit der Selbstbestimmung ab. Das Recht auf Selbstbestimmung wird als das Recht des Einzelnen angesehen, die Grenze zwischen sich und seiner Umwelt zu ziehen. Privatsphäre bedeutet für ihn das Recht jedes Einzelnen, selbst zu entscheiden, was sein eigenes Schicksal sein wird, was er mit sich selbst, seinem Körper und dem Wissen über diese tun wird. ${ }^{9}$

Im Allgemeinen definiert László Székely die Privatsphäre als den innersten Raum des Menschen, in dem er sich ohne Einschränkungen frei ausdrücken kann. ${ }^{10}$

6 Siehe z. B.: KALVEN, JR Harry: The Right of Privacy in Tort Law - Were Warren and Brandeis Wrong? https://chicagounbound.uchicago.edu/cgi/viewcontent.cgi?article=134 22\& context=journal_articles (abgerufen 17. 8. 2020); GLANCY, Dorothy J.: The invention of the right to privacy. Arizona Law Review, 1979/1; SCHWARTZ, Paul M. - PEIFER, Karl Nikolaus: Prosser's Privacy and the german right of personality: Are Four privacy torts better than one unitary concept? California Law Review, 2010/6; SCHOEMAN, Ferdinand D.: Privacy: Philosophical Dimensions of Privacy: An Anthology, Cambridge University Press, Cambridge, 1984; HIRSCH, Dennis D.: Privacy, Public Goods and the Tragedy of the Trust Commons: A Response to Professors Fairfield and Engel, https://papers.ssrn.com/sol3/ papers.cfm?abstract_id=2783933 (abgerufen am 14. 10. 2020).

7 RossiTer, Clinton: Constitutional Dictatorship. 1948, 76.

8 Prosser, William L.: Privacy, California Law Review, 1960. 383.

9 SzABÓ Máté Dániel: Kísérlet a privacy meghatározására a magyar jogrendszer fogalmaival. Infotárs, 2005/5, 46

10 SzÉKELY László: A személyiségi jogok hazai elmélete. A forrásvidék. In medias res, 2012/1, http://real.mtak.hu/108591/1/media-tudomany-szekely-laszlo-a-szemelyisegi-jogok-hazai-elmelete-a-forrasvidek-labady-tamas-cikk-12.pdf (abgerufen am 20. 7. 2020). 
Nach Attila Menyhárd umfasst der Schutz des Rechts auf Privatsphäre das Recht auf körperliche, geistige und soziale Integrität. ${ }^{11}$

Laut Tamás Fézer hat der Schutz der Privatsphäre eine psychische und eine physische Seite. ${ }^{12}$ Die physische Seite ist im Wesentlichen eine Verletzung der Rechte in Bezug auf eine bestimmte Sache, die in der Realität erscheint, die eine Manifestation der Privatsphäre einer Person ist und als ihr Raum dient. Dies könnte zum Beispiel den Schutz der privaten Wohnung oder das Recht auf Achtung des persönlichen Raums umfassen. Die emotionale Dimension der Privatsphäre kann insbesondere der Schutz der Vertraulichkeit, der Schutz vor Bild- und Tonaufnahmen sowie Datenschutzrechte sein. Hierbei handelt es sich um Rechte, die zwar in vielen Fällen in physischer Form, jedoch mit der Person verbunden sind und Informationen über die betreffende Person liefern. ${ }^{13}$

Aus jedem der oben beschriebenen Beispiele können wertvolle Schlussfolgerungen gezogen werden, um den Inhalt des Rechts auf Privatsphäre zu bestimmen.

Aus meiner Sicht kann eine allgemeine Definition des Rechts auf Privatsphäre nicht gegeben werden. Ich halte den Anspruch, eine Definition festzulegen, nicht einmal für wünschenswert, da der Begriff mit einer engeren Definition seine allgemeine, lückenfüllende Rolle verlieren könnte.

Das Recht auf Privatsphäre könnte vielmehr als ein Recht betrachtet werden, das aufgrund der Menschenwürde und des Rechts auf individuelle Selbstbestimmung das ,Recht, allein gelassen zu werden “ bedeutet, sich von anderen (sowohl körperlich als auch geistig) fernzuhalten und Informationen über einen selbst zu löschen. Dieses Recht muss in vertikaler Richtung, d. h. in der Beziehung zwischen Staat und Bürger, und auch horizontal in der Beziehung zwischen einzelnen Personen bestehen. Der Rahmen des ersteren und die Garantien seines Schutzes werden durch internationale Menschenrechtsinstrumente und Verfassungsregeln festgelegt, während der horizontale Geltungsbereich unter der Schirmherrschaft des Privatrechts steht.

Die Rechtsprechung spielt eine Schlüsselrolle bei der Untersuchung bestimmter Bereiche des Rechts auf Privatsphäre. Es gilt insbesondere die wichtige Frage zu beantworten, ob das Recht auf Privatsphäre unabhängig oder nur innerhalb der individuellen Persönlichkeitsrechte ausgeübt werden kann. Meiner Ansicht nach kann der Privatsphäre im weitesten Sinne den Schutz der Privatwohnung, der Kommunikation, des Rechts am eigenen Bild, der Ehre, des Ansehens und der personenbezogenen Daten umfassen, sofern die Verletzung deren einschließlich die Verletzung des Rechts ,,allein gelassen zu werden “(des inneren Kerns von menschliche Intimität) beinhaltet.

11 MENYhÁRD Attila: A magánélethez való jog elméleti alapjai. In Medias Res, 2014/2, 55.

12 FÉZER Tamás: A privátszféra polgári jogi védelmének alapkérdései. http://www.debrecenijogimuhely.hu/archivum/1_2_2014/a_privatszfera_polgari_jogi_vedelmenek_alapkerdesei/ (abgerufen 22. 8. 2020).

13 Ebd. 


\section{GeSETZliche ReGELUNGEN}

Es gibt drei Regelungsebenen für soziale Netzwerke:

- die supranationale Ebene,

- die einschlägigen Vorschriften der Mitgliedstaaten und

- die Richtlinien der sozialen Netzwerke.

Die erste Regelungsebene ist die supranationale Ebene des öffentlichen Rechts, für die mehrere EU-Vorschriften erwähnt werden sollten. Die Plattformen werden teilweise durch die Medienregulierung, ${ }^{14}$ das E-Commerce-Recht, ${ }^{15}$ Vertrags- und Verbraucherrecht ${ }^{16}$ Datenschutzrecht ${ }^{17}$ und Wettbewerbsrecht ${ }^{18}$ abgedeckt. In Bezug auf das hier behandelte Thema ist die Richtlinie 2000/31/EG des Europäischen Parlaments und des Rates (im Folgenden: Richtlinie, Eker-Richtlinie), d. h. die Richtlinie über den elektronischen Geschäftsverkehr, deren Regelungen in das Gesetz CVIII von 2001 über den elektronischen Geschäftsverkehr in das ungarische Recht umgesetzt wurden, besonders hervorzuheben. Diese Richtlinie und das einschlägige innerstaatliche Recht sind hierfür relevant, da Betreiber sozialer Netzwerke als Vermittlungsdienstleister definiert sind und verbindliche Anforderungen für die Entfernung illegaler Inhalte erhalten, die auf den von ihnen betriebenen Plattformen veröffentlicht werden, sowie Regeln für ihre Haftung bei Nichterfüllung dieser Verpflichtungen.

Die zweite Regelungsebene ist die nationale Regulierung der Mitgliedstaaten, die zum Beispiel Folgendes umfasst:

- das Grundgesetz Ungarns

- Gesetz V von 2013 über das Bürgerliche Gesetzbuch

- Gesetz II von 2012 über das Arbeitsgesetzbuch

14 Richtlinie 2010/13/EU des Europäischen Parlaments und des Rates vom 10. März 2010 zur Koordinierung bestimmter Rechts- und Verwaltungsvorschriften der Mitgliedstaaten über die Bereitstellung audiovisueller Mediendienste (Richtlinie über audiovisuelle Mediendienste).

15 Richtlinie 2000/31/EG des Europäischen Parlaments und des Rates vom 8. Juni 2000 über bestimmte rechtliche Aspekte der Dienste der Informationsgesellschaft, insbesondere des elektronischen Geschäftsverkehrs, im Binnenmarkt („Richtlinie über den elektronischen Geschäftsverkehr"); das Gesetz CVIII von 2001 über den elektronischen Geschäftsverkehr.

16 Richtlinie 2005/29/EG des Europäischen Parlaments und des Rates vom 11. Mai 2005 über unlautere Geschäftspraktiken im binnenmarktinternen Geschäftsverkehr zwischen Unternehmen und Verbrauchern und zur Änderung der Richtlinie 84/450/EWG des Rates, der Richtlinien 97/7/EG, 98/27/EG und 2002/65/EG des Europäischen Parlaments und des Rates sowie der Verordnung (EG) Nr. 2006/2004 des Europäischen Parlaments und des Rates (Richtlinie über unlautere Geschäftspraktiken); Gesetz V von 2013 über das Bürgerliche Gesetzbuch.

17 Verordnung (EU) 2016/679 des Europäischen Parlaments und des Rates vom 27. April 2016 zum Schutz natürlicher Personen bei der Verarbeitung personenbezogener Daten, zum freien Datenverkehr und zur Aufhebung der Richtlinie 95/46/EG (DatenschutzGrundverordnung); Gesetz CXII von 2011 über das Recht auf Informationelle Selbstbestimmung und Informationsfreiheit.

18 Vertrag über die Arbeitsweise der Europäischen Union Artikel 101-102. 
- Gesetz CXII von 2011 über das Recht auf Informationelle Selbstbestimmung und Informationsfreiheit

- Gesetz LIII von 2018 über den Schutz der Privatsphäre

- Gesetz CLV von 1997 über den Verbraucherschutz

- Gesetz C von 2012 über das Strafgesetzbuch

Die internen Richtlinien einer bestimmten sozialen Website sind die dritte Ebene, zum Beispiel:

- Nutzungsbedingungen

- Datenverwaltungsrichtlinie

- Gemeinschaftsprinzipien

Diese Regelungsebenen und die gesetzlichen Normen auf jeder Stufe veranschaulichen die Komplexität der Regulierung sozialer Medien.

\section{GESETZLICHE HAFTUNG VON BETREIBERN FÜR DIE VERLETZUNG VON PERSÖNLICHKEITSRECHTE}

Nachdem die Grundlagen besprochen wurden, soll nun untersucht werden, wie sich die Haftung von Betreibern sozialer Netzwerke in Bezug auf die Entfernung von Inhalten, die die Privatsphäre verletzen, im ungarischen Recht entwickelt.

Nach $\S 2: 43$ des ungarischen Bürgerlichen Gesetzbuches (nachfolgend: BGB) ${ }^{19}$ können durch in sozialen Medien veröffentlichte Text-, Bild- oder audiovisuelle Inhalte die benannten Persönlichkeitsrechte, die alle wichtigen Elemente der Privatsphäre enthalten (der Schutz der Privatsphäre, der Schutz des guten Rufs, das Briefgeheimnis, das Bankgeheimnis, das Recht am eigenen Bilde, Tonaufnahmen, das Pietätsrecht sowie der Schutz von personenbezogene Daten) verletzt werden. Diese Rechte werden auf Social-Media-Plattformen am häufigsten durch verschiedene Kommentare, Uploads und Beiträge verletzt.

Die primäre rechtliche Verantwortung für Verletzungen der Persönlichkeitsrechte trägt natürlich der Benutzer, der den anstößigen Inhalt veröffentlicht; das Rechtssubjekt, das in seinen Rechte verletzt wird, hat dann die Möglichkeit im Sinne der $\S \S 2: 51-2: 54$ des Bürgerlichen Gesetzbuchs ${ }^{20}$ gegen den Benutzer den Zivilrechtsweg einzuschlagen. Die Verantwortung der Betreiber sozialer Netzwerke wird

19 BGB 2:43. § [Benannte Persönlichkeitsrechte]

„Eine Verletzung der Persönlichkeitsrechte stellt insbesondere Folgendes dar:

a) die Verletzung des Lebens, der körperlichen Unversehrtheit und der Gesundheit;

b) die Verletzung der persönlichen Freiheit, des Privatlebens bzw. der Privatwohnung;

c) die Diskriminierung von Personen;

d) die Verletzung der persönlichen Ehre und die Rufschädigung;

e) die Verletzung des Rechts auf Schutz von Privatgeheimnissen und personenbezogenen

Daten;

f) die Verletzung des Rechts auf Namensführung;

g) die Verletzung des Rechts am eigenen Bild und am gesprochenen Wort."

20 BGB 2:51. § [Von der Schuldhaftigkeit unabhängige Sanktionen] 
in der Eker-Richtlinie festgestellt. In Artikel 12 der Richtlinie heißt es: ,,im Fall eines Dienstes der Informationsgesellschaft, der darin besteht, von einem Nutzer eingegebene Informationen in einem Kommunikationsnetz zu übermitteln, der Diensteanbieter nicht für die automatische, zeitlich begrenzte Zwischenspeicherung verantwortlich ist, die dem alleinigen Zweck dient, die Übermittlung der Information an andere Nutzer auf deren Anfrage effizienter zu gestalten, sofern folgende Voraussetzungen erfüllt sind:

a) Der Diensteanbieter verändert die Information nicht;

b) der Diensteanbieter beachtet die Bedingungen für den Zugang zu der Information;

c) der Diensteanbieter beachtet die Regeln für die Aktualisierung der Information, die in weithin anerkannten und verwendeten Industriestandards festgelegt sind. "21

Diese Bestimmung sieht eine eher „geringfügige“ Haftung der Vermittlungsdienstleister vor. Bei Verstößen gegen verschiedene Persönlichkeitsrechte kann der Betreiber sozialer Netzwerke jedoch auch für die nicht rechtzeitige Entfernung der

„(1) Die Person, deren Persönlichkeitsrechte verletzt wurden, kann aufgrund der Tatsache der Rechtsverletzung - innerhalb der Verjährungsfrist - nach den Umständen des Falles Folgendes fordern:

a) die Feststellung des Erfolgens einer Rechtsverletzung durch ein Gericht;

b) die Einstellung der Rechtsverletzung und das Untersagen einer weiteren Rechtsverletzung durch die rechtsverletzende Person;

c) dass die rechtsverletzende Person eine geeignete Genugtuung leistet und diese auf eigene Kosten eine entsprechende Öffentlichkeit sichern soll;

d) die Einstellung der rechtswidrigen Situation, die Wiederherstellung des Zustands vor der Rechtsverletzung und die Vernichtung der mit der Rechtsverletzung hergestellten Sachen oder die Beseitigung ihres rechtsverletzenden Charakters;

e) dass ihr die rechtsverletzende Person oder ihr Rechtsnachfolger nach den Regeln einer ungerechtfertigten Bereicherung den mit der Rechtsverletzung erzielten materiellen Vorteil abtreten soll."

BGB 2:52. § [Schmerzensgeld]

„(1) Die Person, deren Persönlichkeitsrechte verletzt wurden, kann für die ihr widerfahrenen immateriellen Schäden ein Schmerzensgeld fordern.

(2) Bezüglich der Bedingungen der Verpflichtung zur Zahlung von Schmerzensgeld - insbesondere bei der Bestimmung der zu Schmerzensgeld verpflichteten Person sowie der Art und Weise der Entschuldigung - sind die Regeln der Schadenersatzhaftung anzuwenden, unter der Maßgabe, dass bei der Berechtigung zu Schmerzensgeld außer der Tatsache der Rechtsverletzung kein Nachweis für das Eintreten weiterer Nachteile erforderlich ist.

(3) Die Höhe des Schmerzensgeldes legt das Gericht mit Rücksicht auf die Umstände des Falls - insbesondere die Schwere und den Wiederholungscharakter der Rechtsverletzung, das Ausmaß des Verschuldens bzw. die auf die geschädigte Person und ihr Umfeld ausgeübte Wirkung der Rechtsverletzung - in einem Betrag fest."

BGB $\S 2: 53$ [Schadenersatzhaftung]

„,Wer durch die Verletzung seiner Persönlichkeitsrechte einen Schaden erleidet, kann von der rechtsverletzenden Person nach den Regeln der Haftung für rechtswidrig verursachte Schäden die Erstattung seines Schadens fordern."

21 Richtlinie über den elektronischen Geschäftsverkehr Artikel 12. 
verletzenden Inhalte haftbar gemacht werden. Im ungarischen Rechtssystem wurde das ,Notice and Take Down"-Verfahren im Zusammenhang mit dem Schutz verschiedener Urheberrechte erklärt, die auch bei Verletzungen von Persönlichkeitsrechten gelten können. Für den Fall, dass ein Benutzer der Website das Gefühl hat, dass sein Recht auf Privatsphäre verletzt wurde und dies dem Betreiber der Website meldet, ist die Website daher verpflichtet, die Meldung zu prüfen und eine Entscheidung darüber zu treffen. Wenn ein Verstoß festgestellt wird, ist die Website verpflichtet, den Inhalt zu entfernen. Geschieht dies nicht, kann die Website selbst haftbar gemacht werden. Es bestehen zwei Haftungsmöglichkeiten:

- Ein zivilrechtlicher Anspruch der von dem Inhalt verletzten Person auf Entfernung des Inhalts besteht bei jeder nicht oder nicht rechtzeitig durchgeführten inhaltliche Untersuchung.

- Sonstige rechtliche Haftungen für Verstöße, die von einer zuständigen Stelle (Behörde) oder einem Gericht festgestellt wurden, bestehen, sofern das Rechtssystem des jeweiligen Staates dies zulässt.

Der Gerichtshof der Europäischen Union (EuGH) hat sich mehrfach mit der Haftung von Vermittlungsdienstleistern befasst. Als Beispiel argumentierte der EuGH im Urteil Google France SARL und Google Inc. gegen Louis Vuitton Malletier SA von $2010^{22}$, dass um zu entscheiden, ob eine Haftungsbeschränkung auf HostingAnbieter angewendet werden soll oder nicht, sollte der folgende Absatz der EkerRichtlinie angenommen werden: ,, diese Tätigkeit [des Hosting-Anbieters] ist rein technischer, automatischer und passiver Art, was bedeutet, dass der Anbieter eines Dienstes der Informationsgesellschaft weder Kenntnis noch Kontrolle über die weitergeleitete oder gespeicherte Information besitzt. " 23

Darüber hinaus wurde in der Mitteilung der Europäischen Kommission vom 28. September 2017 die Bedeutung der Bekämpfung illegaler Inhalte und parallel dazu die zunehmende Verantwortung von Online-Plattformen hervorgehoben. Die Mitteilung ist nicht bindend, sondern dient lediglich als Leitfaden für Online-Plattformbetreiber, um bestimmte illegale Inhalte proaktiv zu erkennen und zu entfernen (unzugänglich zu machen).

Anschließend verabschiedete die Kommission im März 2018 eine Empfehlung für wirksame Maßnahmen im Umgang mit illegalen Online-Inhalten. Obwohl keines dieser beiden Dokumente bindend ist, haben sie den Diskurs zwischen den EU-Mitgliedstaaten über die Verantwortlichkeiten der Betreiber von Social-Media-Webseiten gefördert.

\section{BEISPIELE}

Es gibt einige lehrreiche Fälle zur Haftung von Vermittlungsdienstleistern aus der Rechtsprechung des Europäischen Gerichtshofs für Menschenrechte (EGMR) und

22 Beitritt zu den Rechtssachen C-236/08 und C-238/08 vor dem Gerichtshof der Europäischen Union.

23 Richtlinie über den elektronischen Geschäftsverkehr Artikel 43. 
aus der innerstaatlichen Rechtsprechung. In Bezug auf Kommentare war der erste Meilenstein die Entscheidung des EGMR in der Rechtssache Delphi AS gegen Estland aus dem Jahr 2013. ${ }^{24}$ Es wurde entschieden, dass Internet-Newsportale für verletzende Kommentare haftbar gemacht werden können. Die Bedeutung des Falles war in Bezug auf Haftungsfragen herausragend, da das Gericht die Verantwortung für bearbeitete Online-Inhalte weiter interpretierte als die Richtlinie. Der Fall basierte auf einem Zeitungsartikel, der auf dem größten estnischen Internet-Newsportal Delfi erschien. Dem Artikel zufolge gefroren die Gewässer vor der Küste Estlands im Winter so stark, dass es zweckmäßiger erschien, sich einigen Inseln auf eisgeführten Straßen zu nähern, diese Eisstraßen jedoch nicht geöffnet werden konnten. Als Grund wurde angegeben, dass eine Reederei, die Fähren zwischen dem Festland und den Inseln anbietet, ihre Route so geändert hatte, dass sich das Eis nicht genug verdicken konnte, was den Verkehr unsicher machte. Es gab dabei auf der Internetseite die Möglichkeit den Artikel zu kommentieren, was eine große Anzahl von Lesern auch tat. Unter den Kommentaren gab es vermehrt Beiträge, die die Reederei und einen ihrer Hauptaktionäre scharf kritisierten. Die Reederei forderte daraufhin die Website auf, die beleidigenden Bemerkungen zu entfernen, dem Delfi auch nachkam. Später reichte das betreffende Unternehmen jedoch eine Zivilklage gegen Delfi ein, in deren Verlauf estnische Gerichte die Website zu Entschädigungszahlungen verurteilten, obwohl bis dahin alle Kommentare, die die Datenschutzrechte verletzten, entfernt worden waren. Das InternetNewsportal legte daraufhin Berufung beim EGMR ein, der entschied, dass die Meinungsfreiheit im vorliegenden Fall nicht unangemessen verletzt worden sei.

In seinem Urteil stellte der EGMR unter anderem fest, dass Delfi nicht nur von dem Artikel selbst profitierte, sondern auch von der Kommentarfunktion. Nach Angaben des Gerichts hätte Delfi die negativen Kommentare, die ihr Artikel hervorrief, voraussehen und ihr Kommentarsystem entsprechend anpassen müssen. Die Zeitung hätte, nach dem Urteil des EGMR, verhindern sollen, dass diffamierende Aussagen veröffentlicht werden. Dabei war auch die Tatsache von besonderer Bedeutung, dass die Website die Möglichkeit des Kommentierens nicht von einer Registrierung abhängig machte, wodurch die Möglichkeit, Kommentatoren zur Rechenschaft zu ziehen und damit die primäre rechtliche Haftung durchzusetzen, praktisch unmöglich war. ${ }^{25}$

Wenden wir uns der Praxis des ungarischen Verfassungsgerichts (VG) zu, wobei wir nur ein Beispiel nennen: In der Entscheidung 9/2014. (V. 30.) $A B$ untersuchte das VG die Frage der Haftung für die Entfernung von Kommentaren, die das Recht auf Privatsphäre verletzen. In seiner Entscheidung stellte das Verfassungsgericht fest, dass ein signifikanter Unterschied zwischen (Inhalts-)Diensten, die vom Betreiber einer Internetseite herausgegeben werden und eine inhaltliche Einheit bilden, und dem sog. Web 2.0, d. h. sozialen Netzwerken und reinen Meinungsseiten (z. B. Facebook, Blogosphäre, etc.) bestehe. Letztere haben keinen Redakteur und daher auch keine informative oder ähnliche Funktion: Ihr Ziel ist es, den Gedankenaustausch zwischen den Mitgliedern der Internet-Gemeinschaft und die Kommunikation im

24 EGMR, 10. 10. 2013, Rs. C-345/17, www.curia.eu (Delfi v. Estonia).

25 Ebd. 
Allgemeinen zu ermöglichen. ${ }^{26}$ Letztendlich hat das VG eine Haftung Facebooks für Beiträge unter Facebook-Profilen oder -Seiten ausgeschlossen, da diese keine Inhalte darstellen, die Internetnutzern öffentlich zugänglich sind, sondern nur ,Freunden “. In der Realität ist dieser Ansatz jedoch besorgniserregend, weshalb die diesbezügliche Position der Kurie (Oberster Gerichtshof Ungarns) erwähnt werden sollte: ,„zusätzlich zu der Person, die den Kommentar gepostet hat, wird [der Betreiber] Teil der Erklärung oder der Tatsachenbehauptung sein, genauso wie die Pressestelle den Inhalt der Leserbriefe im Presseprodukt kommuniziert". ${ }^{27}$ Wenn der Kommentar diffamierend war, impliziert dies direkt die Verantwortung des Betreibers.

Neben den Kommentaren ist auch das Thema gezielte Werbung in sozialen Medien und deren Verantwortung unter dem Gesichtspunkt des Schutzes der Privatsphäre hervorzuheben, da die meisten dieser Anzeigen auf der Verfolgung der Benutzeraktivität auf der Website basieren. Daher kann die ,Profilerstellung “ und Zuordnung eines Benutzers durch eine soziale Website ein wichtiges Anliegen für den Schutz der Privatsphäre sein. Es ist äußerst besorgniserregend, dass sich die Datenerfassung auf nicht veröffentlichte Meinungen erstrecken kann, die in Gedankenform bleiben. Ein Beispiel hierfür ist die Einbeziehung der Analyse ungesendeter oder unveröffentlichter Nachrichten und Inhalte in die Erstellung des Benutzerprofils. Auf diese Weise wird die nicht gesendete Nachricht nicht vollständig geheim gehalten, was in der Offline-Welt normalerweise selbstverständlich ist. In Bezug auf solche Werbung können Benutzer eine mögliche Verletzung der Privatsphäre der Website melden, die diese im Rahmen des oben erwähnten Verfahrens zur Meldung und Entfernung untersucht. Damit ist die Plattform gezwungen, gleichzeitig eine redaktionelle und juristische Rolle zu spielen, über die Löschung oder weitere Verfügbarkeit des Inhalts und damit auch über dessen Rechtmäßigkeit zu entscheiden. Diese Regelung wirft offensichtlich Bedenken auf. Wenn die Plattform den beanstandeten Inhalt nicht entfernt, wird sie selbst verklagbar.

\section{FAZIT}

Insgesamt haben soziale Netzwerke als freier Markt für Ideen einen enormen Einfluss. Als Ausgangspunkt für die Haftung von Social-Media-Plattformen können die Regeln des ,,Notice and Take Down "-Verfahrens verwendet werden, für die auch die Rechtsprechung einen zunehmenden Trend zeigt. Ausgangspunkt für die Haftung der Seiten ist das in der Eker-Richtlinie geregelte „Notice and Take Down“-Verfahren, zu dem auch die Rechtsprechung tendiert. Als freier öffentlicher Raum für Meinungen sollten sie auch den Schutz der Privatsphäre gewährleisten, die sich als eine komplexe, von Fall zu Fall unterschiedliche Aufgabe für Rechtsanwender erwiesen kann.

Die Haftung von Betreibern sozialer Netzwerke ist ein scharfes Querschnittsthema von öffentlichem Interesse und die Festlegung ihres Rahmens ist eine wichtige gesetzgeberische Aufgabe, die übernommen werden muss.

26 Entscheidung Nr. 9/2014 (V. 30.) des Verfassungsgerichts (61).

27 Oberster Gerichtshof. Nr. Pfv. IV. 20.794/2016. 


\section{LITERATURVERZEICHNIS}

[1] FÉZER Tamás: A privátszféra polgári jogi védelmének alapkérdései. Debreceni Jogi Mühely, 2014/1-2, http://doi.org/10.24169/DJM/2014/1-2/5. http://www.debrecenijogimuhely.hu/archivum/1_2_2014/a_privatszfera_polgari_jogi_vedelmenek_alapkerdesei/ (abgerufen am 22. 8. 2020).

[2] Glancy, Dorothy J.: The invention of the right to privacy. Arizona Law Review, 1979/1.

[3] HIRSCH, Dennis D.: Privacy, Public Goods and the Tragedy of the Trust Commons: A Response to Professors Fairfield and Engel. https://papers.ssrn. com/sol3/papers.cfm?abstract_id $=2783933$ (abgerufen am 14. 10. 2020).

[4] KALVEN JR, Harry: The Right of Privacy in Tort Law - Were Warren and Brandeis Wrong? https://chicagounbound.uchicago.edu/cgi/viewcontent.cgi? article $=13422 \&$ context $=$ journal_articles (abgerufen am 17. 8. 2020).

[5] MENYHÁRD Attila: A magánélethez való jog elméleti alapjai. In Medias Res. $2014 / 2$.

[6] Prosser, William L.: Privacy. California Law Review, 1960, 383.

[7] Rossiter, Clinton: Constitutional Dictatorship. Princeton University Press, 1948.

[8] Schoeman, Ferdinand D.: Privacy: Philosophical Dimensions of Privacy: An Anthology. Cambridge University Press, Cambridge, 1984.

[9] SChwARTZ, Paul M. - PfeIFER, Karl Nikolaus: Prosser's Privacy and the german right of personality: Are Four privacy torts better than one unitary concept? California Law Reviwev, 2010/6.

[10] SZABÓ Máté Dániel: Kísérlet a privacy meghatározására a magyar jogrendszer fogalmaival. Infotárs, 2005/5.

[11] SzÉKELY László: A személyiségi jogok hazai elmélete. A forrásvidék. In Medias Res, 2012/1. http://real.mtak.hu/108591/1/media-tudomany-szekely-laszloa-szemelyisegi-jogok-hazai-elmelete-a-forrasvidek-labady-tamas-cikk-12.pdf (abgerufen am 20. 7. 2020).

[12] WARREN, Samuel- BRANDEIS, Luis: The Right to privacy. Harvard Law Review, 15. 12. 1890.

[13] ZHAO, Shanyang: Internet Use and Social Capital. Paper presented at the annual meeting of the American SociologicalAssociation, Marriott Hotel, Loews Philadelphia Hotel, Philadelphia, PA, 12. 8. 2005, http:/www.allacademic.com/ one/www/research/index.php?cmd=Download+Document\&key=unpublished manuscript\&file_index $=1 \&$ pop_up=true\&no_click_key=true\&attachment_ style $=$ attachment $\&$ PHPSESSID $=$ cad89aaa $7 \overline{\mathrm{e}} 5888 \overline{2} \mathrm{ff} 54 \mathrm{deba} 93 \mathrm{~d} 04 \mathrm{~d} 2 \mathrm{f} 9$ (abgerufen am 11. 12.20). 